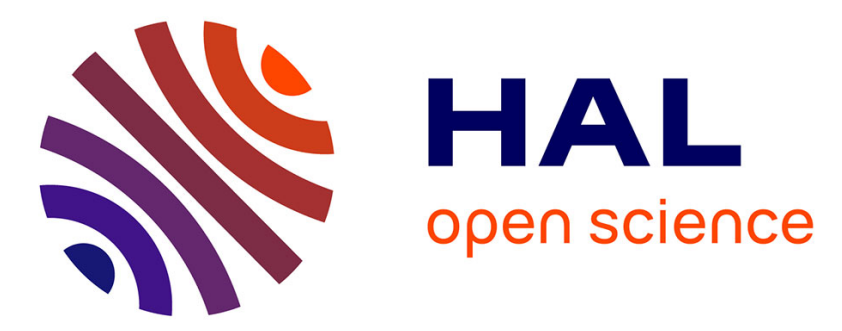

\title{
Collisions between spinning and nonspinning co-axial three-dimensional Ginzburg-Landau solitons
}

Dumitru Mihalache, D. Mazilu, F. Lederer, Hervé Leblond, Boris Malomed

\section{To cite this version:}

Dumitru Mihalache, D. Mazilu, F. Lederer, Hervé Leblond, Boris Malomed. Collisions between spinning and nonspinning co-axial three-dimensional Ginzburg-Landau solitons. The European Physical Journal Special Topics, 2009, 173 (1), pp.245-254. 10.1140/epjst/e2009-01077-7 . hal-03423788

\section{HAL Id: hal-03423788 \\ https://univ-angers.hal.science/hal-03423788}

Submitted on 10 Nov 2021

HAL is a multi-disciplinary open access archive for the deposit and dissemination of scientific research documents, whether they are published or not. The documents may come from teaching and research institutions in France or abroad, or from public or private research centers.
L'archive ouverte pluridisciplinaire HAL, est destinée au dépôt et à la diffusion de documents scientifiques de niveau recherche, publiés ou non, émanant des établissements d'enseignement et de recherche français ou étrangers, des laboratoires publics ou privés. 


\title{
Collisions between spinning and nonspinning co-axial three-dimensional Ginzburg-Landau solitons
}

\author{
D. Mihalache ${ }^{1, a}$, D. Mazilu ${ }^{1}$, F. Lederer ${ }^{2}$, H. Leblond ${ }^{3}$, and B.A. Malomed ${ }^{4}$
}

1 Horia Hulubei National Institute for Physics and Nuclear Engineering (IFIN-HH), 407 Atomistilor, 077125 Magurele-Bucharest, Romania

2 Institute of Solid State Theory and Theoretical Optics, Friedrich-Schiller Universität Jena, Max-Wien-Platz 1, 07743 Jena, Germany

3 Laboratoire POMA, CNRS FRE 2988, Université d'Angers, 2 Bd. Lavoisier, 49045 Angers Cedex 01, France

4 Department of Physical Electronics, Faculty of Engineering, Tel Aviv University, Tel Aviv 69978, Israel

\begin{abstract}
We report results of the first analysis of collisions between stable fundamental (alias spinless) and vortical (spinning) three-dimensional dissipative solitons in a model of a laser cavity. The systematic analysis is carried out for values $S=1$ and $S=2$ of the vorticity of the latter soliton. With the increase of the collision momentum, $\chi$, the same generic scenarios are observed in either case: merger into a single fundamental soliton at both small and relatively large values of $\chi$, and the formation of two fundamental solitons in an intermediate interval of variation of the collision momentum $\chi$. At very large values of $\chi$, the collision seems quasi-elastic, but the vortex soliton eventually splits into two nonspinning fragments.
\end{abstract}

\section{Introduction}

The class of complex Ginzburg-Landau (CGL) equations constitute a mathematical framework for modeling nonlinear dynamics in situations close to pattern-forming transitions in various dissipative media, such as optical cavities, viscous fluid flows and thermal convection, reactiondiffusion mixtures, and others [1]. Localized (solitary) pulses, alias dissipative solitons (DSs), constitute an important type of patterns which occur in these media and can be effectively described by the CGL equations [2-4]. The search for stable DS solutions is a challenge in modeling two- and three-dimensional (2D and 3D) media, because of the possibility of the critical or supercritical collapse, in the 2D and 3D geometry, respectively, under the action of the self-focusing cubic nonlinearity. In addition to that, multidimensional localized states with intrinsic vorticity, i.e., vortex solitons, are prone to instability against azimuthal perturbations that tend to split them $[5,6]$.

It is well known that a physically relevant model which may support stable DSs in any dimension is provided by the CGL equation with the cubic-quintic (CQ) nonlinearity. This equation was originally introduced by Petviashvili and Sergeev [7] in the 2D form, with the objective to generate vortical DSs. In the 1D version of the same model, solitary-pulse solutions and their stability were then investigated in detail [8]. Nonlinear optical media that feature the CQ response include chalcogenide glasses [9], some organic materials [10], aqueous colloids [11], and dye solutions [12].

\footnotetext{
a e-mail: Dumitru.Mihalache@nipne.ro
} 
Actually, stable 2D vortical solitons, with topological charge (vorticity) $S=1$ and 2 , were constructed in the framework of the CQ CGL equation in Refs. [13]. Stable fundamental $(S=0)$ 3D localized states were found as spatiotemporal DSs in optical models based on equations of the same type [14-17]. 3D double-soliton complexes, including rotating ones [18], have been found too. Finally, 3D vortex solitons with $S=1,2$, and 3 have been obtained recently, as solutions to the CQ CGL equation [19-21]. The stability of the latter solutions was analyzed in terms of the growth rates of perturbation eigenmodes, and verified in direct simulations. It is also relevant to mention that the dynamics of weakly nonstationary ring-shaped $2 \mathrm{D}$ and $3 \mathrm{D}$ soliton clusters was studied in some detail, in various conservative and dissipative settings [22-27].

Once stable 3D vortex solitons are available, a problem of obvious interest is to consider collisions between them. Recently [28], we have investigated collisions between two 3D vortex solitons with identical vorticities $S_{1}=S_{2} \equiv S(S=1,2)$ in a coaxial configuration, as well as collisions between counter-rotating vortices, with $S_{1}=-S_{2}=1$ or 2 [29]. This was done in the framework of the following CQ CGL equation in three dimensions [19,20]:

$$
\begin{gathered}
\quad i U_{z}+(1 / 2-i \beta)\left(U_{x x}+U_{y y}\right)+(D / 2) U_{t t} \\
+\left[i \delta+(1-i \varepsilon)|U|^{2}-(\nu-i \mu)|U|^{4}\right] U=0 .
\end{gathered}
$$

In term of nonlinear optics, $U$ is the local amplitude of the electromagnetic wave in the bulk medium which propagates along axis $z$, the transverse coordinates are $x$ and $y$, while the temporal variable is $t=T-z / V_{0}$, where $T$ is time and $V_{0}$ is the group velocity of the carrier wave. The coefficients which are scaled to be $1 / 2$ and 1 account, respectively, for diffraction in the transverse plane and the self-focusing Kerr nonlinearity, $\beta \geq 0$ is the effective diffusivity in the transverse plane, real constants $\delta, \varepsilon$ and $\mu$ represent, respectively, the linear loss, cubic gain, and quintic loss (the basic ingredients of the CQ CGL equation [7]). Here $\nu \geq 0$ accounts for the self-defocusing quintic nonlinearity, that may compete with the cubic term, according to the experimental observations $[9,10]$, and $D$ is the group-velocity dispersion (GVD) coefficient. Its positive and negative values correspond, severally, to the GVD of the anomalous and normal types.

Equation (1) admits free motion of solitons along axis $z$, and thus makes collisions between them possible [30]. On the contrary to that, free motion in plane $(x, y)$ is impeded by the diffusivity term in Eq. (1), the one with $\beta>0$. As shown in Refs. [19,20], this term is necessary for the stability of vortex DSs, while fundamental solitons, with $S=0$, may be stable at $\beta=0$ as well.

In the general case, analysis of collisions between localized objects in the 3D space is quite involved, requiring heavy simulations of the full 3D equations. In particular, in the framework of the CGL equation (1) with $\beta=0$, where the free motion is possible both in the transverse plane $(x, y)$ and along axis $z$, the collision between zero-spin solitons is controlled by four parameters: relative transverse and axial velocities of the solitons, and two respective aiming parameters (in terms of the optical model, the aiming parameter related to the motion along $z$ is realized as the temporal separation between the solitons when their separation in the $(x, y)$ plane attains a minimum). Examples of collisions between 3D DSs with $S=0$ were reported in Ref. [17]. Outcomes observed in those simulations included fusion of the solitons into a single one (sometimes, it looked as destruction of one of the colliding objects), or quasi-elastic interaction (mutual rebound of the solitons).

As mentioned above, if the colliding solitons carry vorticity, the CGL equation must include the term proportional to the diffusivity parameter $\beta>0$, otherwise the vortex solitons are unstable. Then, as their motion in the transverse plane is suppressed by the diffusivity term, the collision depends on two parameters, viz., the relative velocity in the axial direction and the related aiming parameter, which is the distance between parallel trajectories of the colliding solitons. In a recent work [28], we focused on a practically tractable setting, by choosing the diffusivity coefficient $\beta>0$ and zero aiming parameter, when the $3 \mathrm{D}$ colliding vortex solitons form a coaxial configuration. The analysis is feasible in that case, due to the inherent axial symmetry (nevertheless, the numerical integration of the CGL equation was performed in the Cartesian coordinates, which guarantees that all potentially dangerous perturbations are incorporated, 
including those which may break the axial symmetry). Depending on the collision momentum, $\chi$, we have identified three generic outcomes [28]: merger of the solitons into a single one, at small $\chi$; quasi-elastic interaction, at large $\chi$; and creation of an extra soliton ("soliton birth" $[31])$, in an intermediate region.

In this work we consider a different problem, viz., the collision between a fundamental (nonspinning) soliton, with $S=0$, and another one, with nonzero vorticity $S=1$ or 2 . In this case, one may take the latter (spinning) soliton with zero velocity, and set in motion only the fundamental one, multiplying it by $\exp (i \chi t)$, where $\chi$ is the "kick" parameter. Generic outcomes of the collisions produced by our numerical analysis are merger of the two solitons into one at both small and relatively large values of $\chi$, formation of two fundamental solitons in an intermediate interval of variation of the collision momentum $\chi$, and a quasi-elastic collision at very large $\chi$, followed by a subsequent splitting of the spinning soliton into two spinless fragments.

The rest of this paper is organized as follows. The model equation and setting for the analysis are formulated in Sec. 2, the collisions outcomes are reported in Sec. 3, and the paper is concluded by Sec. 4.

\section{The cubic-quintic Ginzburg-Landau model and stable $3 \mathrm{D}$ vortex solitons}

In the general case, the 3D CGL equation with the CQ nonlinearity takes the form of Eq. (1) [19,20]. All coefficients in Eq. (1) are well-known physical parameters, except for a more "exotic" one, $\beta$. The diffusivity term proportional to $\beta$ appears in a model of laser cavities, where it is generated by the interplay of the dephasing of local polarization in the dielectric medium, cavity loss, and detuning between the cavity's and atomic frequencies [32]. As said above, we will keep $\beta>0$ in Eq. (1), to secure the stability of the vortex solitons.

In the conservative counterpart of the CQ CGL equation, i.e., the nonlinear Schrödinger equation with the $\mathrm{CQ}$ nonlinearity, the quintic term must be self-defocusing (in the $2 \mathrm{D}$ and $3 \mathrm{D}$ geometries), to arrest the collapse induced by the self-focusing cubic term $[5,33]$. However, the self-defocusing sign of the quintic term is not necessary for the stability of the multidimensional DSs, because the collapse is prevented by the stronger effect of the quintic term in the dissipative part of the equation [19]. The sign of the GVD coefficient, $D$, is not crucial either, because the existence of stable fundamental and vortical 3D solitons was demonstrated for both $D>0[19]$ and $D<0[20]$, both cases being relevant to optics $(D<0$, i.e., normal GVD, gives rise to strong phase chirp along the temporal direction in the soliton).

First we look for stationary DS solutions to Eq. (1) in the form of

$$
U(z, x, y, t)=\psi(r, t) \exp (i k z+i S \theta)
$$

where $r$ and $\theta$ are the polar coordinates in plane $(x, y), S \geq 0$ the above-mentioned integer vorticity (the fundamental solitons correspond to $S=0$ ), $k$ a real wave number, and the complex

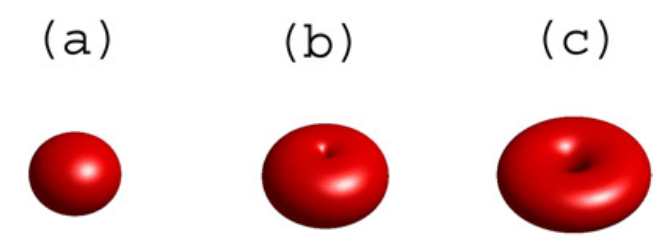

Fig. 1. (Color online) Isosurface plots of intensity $|U(x, y, t)|^{2}$, showing the input solitons: (a) $S=0$, (b) $S=1$, and (c) $S=2$. 


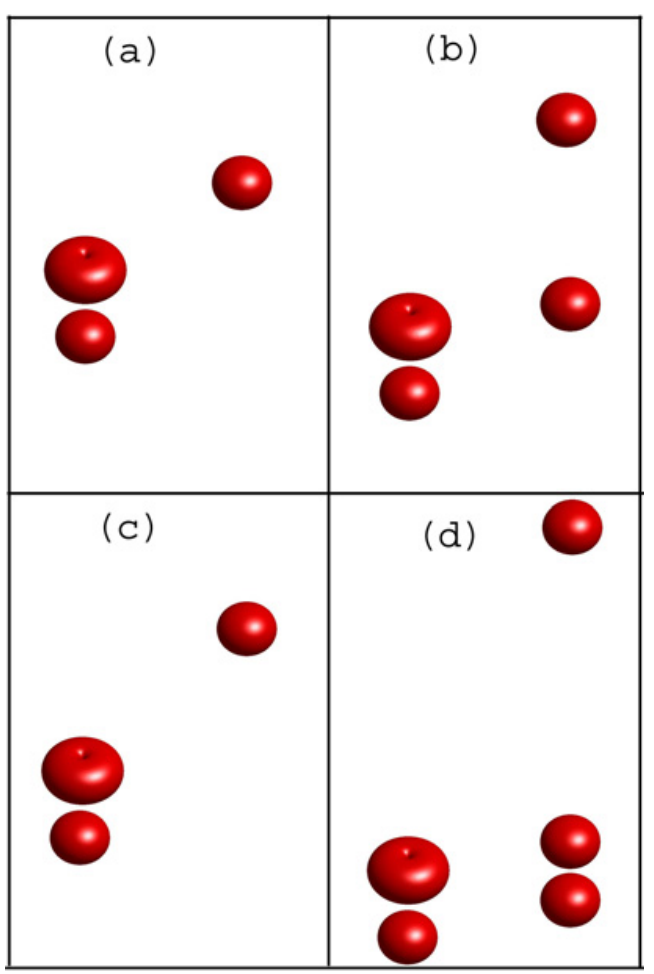

Fig. 2. (Color online) Isosurface plots of intensity $|U(x, y, t)|^{2}$, showing the colliding solitons with vorticities 0 and 1 , and the output set, at different values of the kick parameter: (a) $\chi=0.4$; (b) $\chi=1.5$; (c) $\chi=2$; (d) $\chi=2.5$. The simulations were run on the grid of size $193 \times 193 \times 801$.

function $\psi(r, t)$ obeys the stationary equation,

$$
\begin{aligned}
& \left(\frac{1}{2}-i \beta\right)\left(\psi_{r r}+\frac{1}{r} \psi_{r}-\frac{S^{2}}{r^{2}} \psi\right)+\frac{1}{2} D \psi_{t t} \\
+ & {\left[i \delta+(1-i \varepsilon)|\psi|^{2}-(\nu-i \mu)|\psi|^{4}\right] \psi=k \psi . }
\end{aligned}
$$

Localized solutions to this equation must decay exponentially at $r,|t| \rightarrow \infty$, and must behave as $r^{|S|}$ at $r \rightarrow 0$.

Below, we consider the following set of parameters: $D=1$ (anomalous GVD), $\mu=1, \nu=0.1$, $\delta=0.4$, and $\beta=0.5$. We will consider collisions between stable DSs with vorticities $S=0$, and $S=1,2$. To generate these solitons, we simulated the propagation of zero-vorticity and vortical localized pulses forward in $z$, within the framework of the radial version of Eq. (1), produced by the substitution of $U(z, x, y, t)=\Psi(z, r, t) \exp (i S \theta)$. The following axially symmetric input spatiotemporal field distribution was used for this purpose:

$$
\Psi(0, r, t)=A r^{|S|} \exp \left[-(1 / 2)\left(r^{2} / \rho^{2}+t^{2} / \tau^{2}\right)\right],
$$

with some constants $A, \rho$, and $\tau$. The standard Crank-Nicholson scheme was used for the numerical integration (typically, with transverse and longitudinal stepsizes $\Delta r=\Delta t=0.2$ and $\Delta z=0.01$ ). The nonlinear finite-difference equations were solved with the help of the Picard iteration method, and the resulting linear system was then handled by means of the Gauss-Seidel iterative procedure. To achieve reliable convergence to stationary states, ten Picard and four Gauss-Seidel iterations were usually sufficient. The wave number $(k)$ of the established solution was found as the corresponding value of the $z$-derivative of the phase of $\Psi(z, r, t)$, provided that it became independent on $z, r$ and $t$ up to five significant digits.

In what follows we also fix the value of the cubic gain parameter to $\varepsilon=2.3$, which lies within the stability domain of vortex solitons with vorticities $S=1$ and 2, see Ref. [20]. Note 


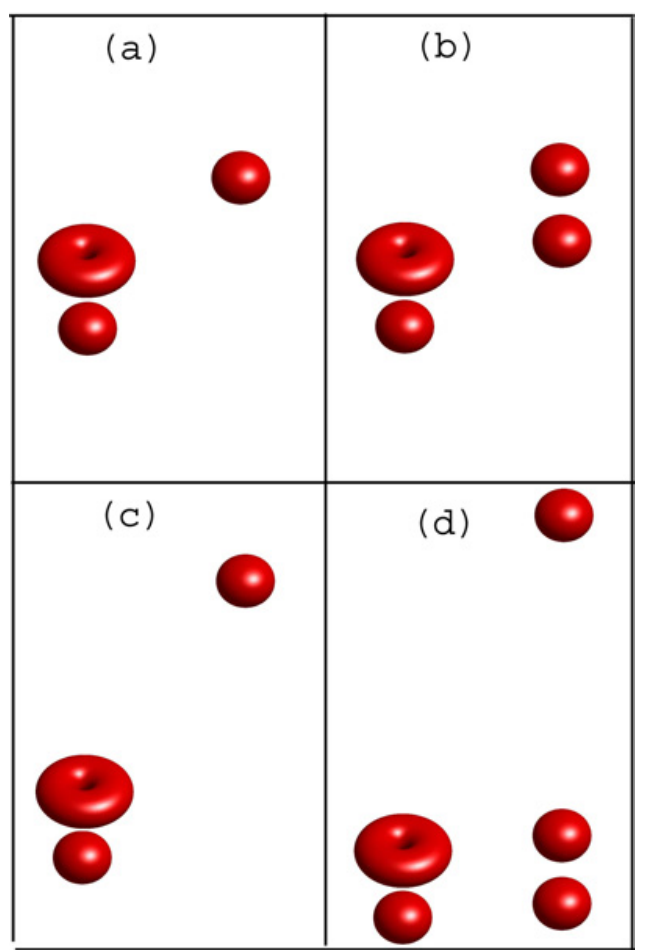

Fig. 3. (Color online) The same as in Fig. 2, but for collisions of solitons with vorticities 0 and 2 : (a) $\chi=0.2$; (b) $\chi=1$; (c) $\chi=2$; (d) $\chi=2.5$. The grid size is $201 \times 201 \times 801$.

that the values of the wave numbers (with five significant digits) are $k=0.44342$ for $S=0$, $k=0.50040$ for $S=1$, and $k=0.50387$ for $S=2$.

Thus, to study generic outcomes of collisions between DSs, one should take a pair of stable solitons with shapes shown in Fig. 1, separated by a large temporal distance $T$. As mentioned above, we will consider collisions between the spinless soliton (with $S=0$ ) and another one, with $S=1$ or 2 .

\section{Collision outcomes}

To solve Eq. (1) with the initial condition (at $z=0$ ) corresponding to collisions between two stable solitons with vorticities $(0, S)$, where $S=1,2$, initially separated by a large temporal distance $T$, a 3D Crank-Nicolson finite-difference scheme was used, with typical transverse and longitudinal stepsizes $\Delta x=\Delta y=\Delta t=0.2$ and $\Delta z=0.01$. Similar to the simulations outlined in the previous section, the resulting nonlinear finite-difference equations were solved using the Picard iteration method, and the ensuing linear system was then dealt with by means of the Gauss-Seidel elimination procedure. To achieve good convergence, ten Picard and four GaussSeidel iterations were typically needed. In most cases, we used 801 discretization points for $t$, while the number of the mesh points for $x$ and $y$ that provided for the required accuracy depended on the vorticity: $193 \times 193$ for $S=1$, and $201 \times 201$ for $S=2$.

Results of systematic simulations of soliton collisions are represented by outcomes observed with the variation of kick parameter $\chi$. For our set of parameters, the $3 \mathrm{D}$ solitons with $S=0,1,2$ (and also with $S=3$ ) are all stable [19,20], being characterized by the following values of the energy (alias norm),

$$
E \equiv 2 \pi \int_{0}^{\infty} r d r \int_{-\infty}^{+\infty} d t|\psi(r, t)|^{2}
$$

$E(S=0) \approx 52, E(S=1) \approx 171$, and $E(S=2) \approx 310$. 

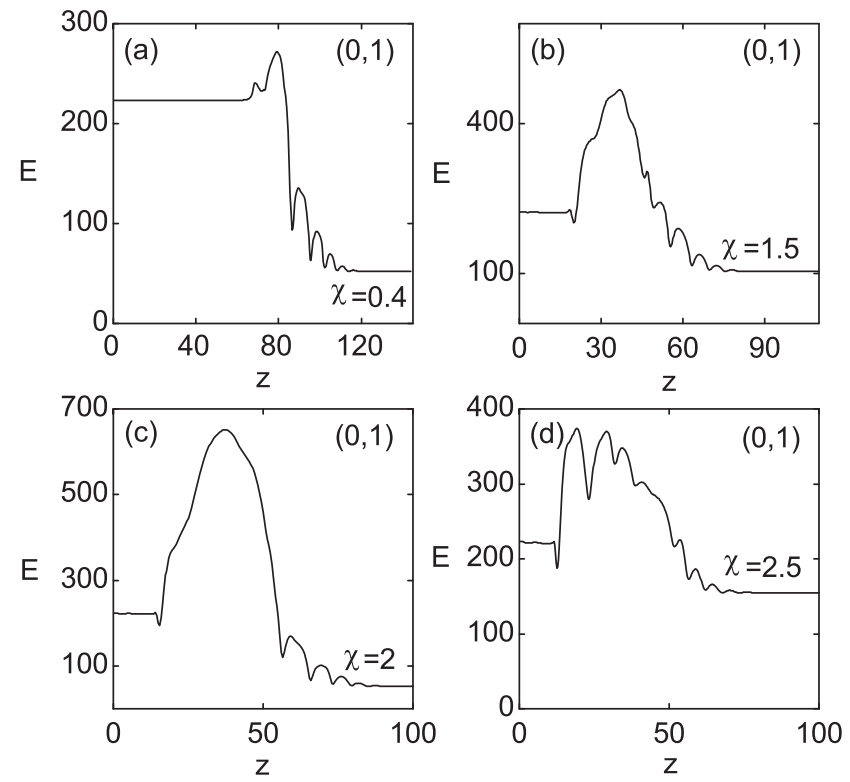

Fig. 4. The evolution of the total energy in the collision of $(0,1)$ solitons at different values of the kick parameter: (a) $\chi=0.4$; (b) $\chi=1.5$; (c) $\chi=2$; (d) $\chi=2.5$.
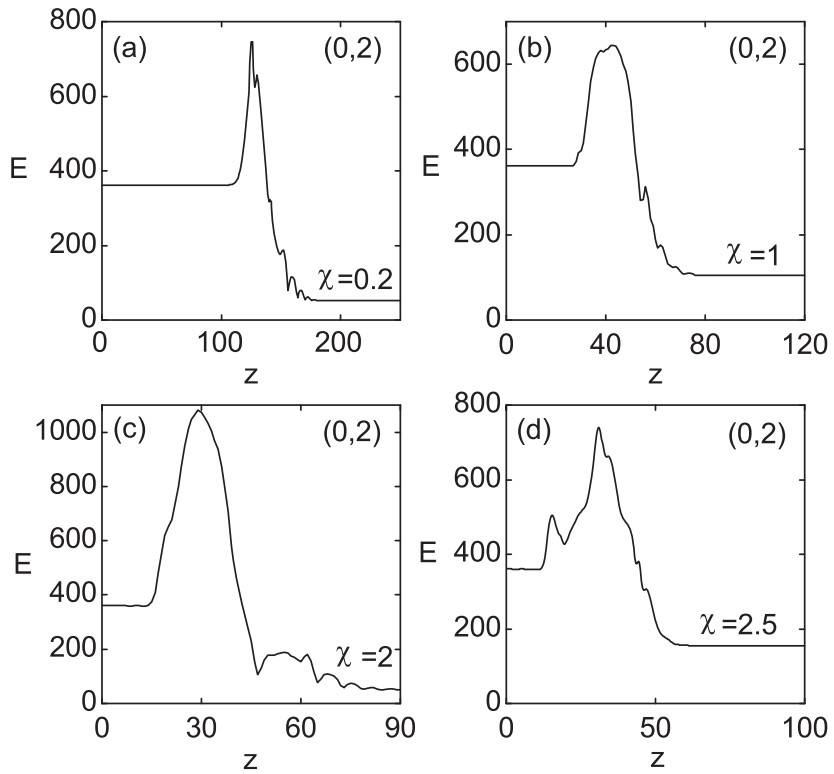

Fig. 5. The same as in Fig. 4 , but for the collision of $(0,2)$ solitons: (a) $\chi=0.2$; (b) $\chi=1$; (c) $\chi=2$; (d) $\chi=2.5$.

In the following we report the outcomes of collisions for DS pairs with vorticities $(0,1)$ and $(0,2)$. Gradually increasing the initial kick $\chi$, we have observed the following outcomes (the initial separation was typically $T=30$, but variation of $T$ did not affect the results):

(a) Merger of the two solitons into a single fundamental soliton, with $S=0$, at small values of $\chi$. The velocity of the emerging fundamental soliton is larger than the velocity of the initial one.

(b) Generation of two fundamental solitons with different velocities: while the initial fundamental soliton reduces its velocity after the collision, a new soliton with $S=0$ has a very small velocity. 

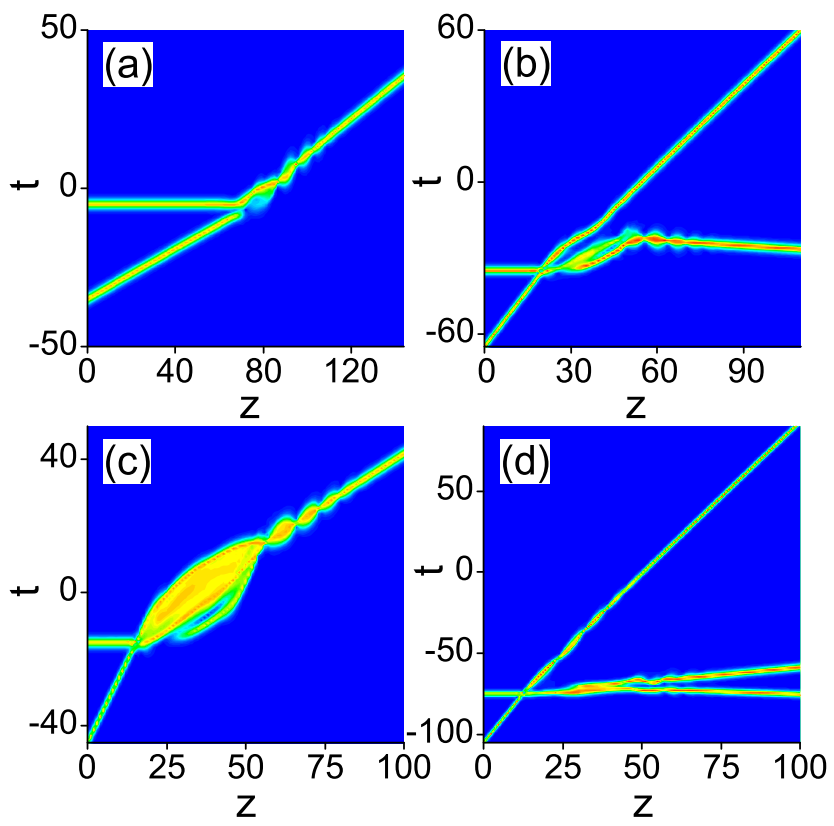

Fig. 6. (Color online) Contour plots displaying the evolution of field $|U|$ in plane $(t, z)$, for the collision scenarios of $(0,1)$ solitons at different values of kick $\chi$ : a) $\chi=0.4$; (b) $\chi=1.5$; (c) $\chi=2$; (d) $\chi=2.5$.

(c) Merger of the two solitons into a single fundamental one, at relatively large values of $\chi$. On the contrary to case (a), the the emerging fundamental soliton is slower than its initial counterpart.

(d) Quasi-elastic collision of the spinless and spinning solitons, followed by subsequent splitting of the perturbed spinning soliton into two spinless fragments moving with opposite velocities, at very large values of $\chi$.

These generic outcomes are illustrated by Figs. 2 and 3, which display, by dint of isosurfaces of the local intensity, $|U(x, y, t)|^{2}$, the initial set of the colliding DSs, and the outcome sets observed long after the collision.

These collision scenarios are further illustrated by Figs. 4 and 5 which show the total energy of the colliding solitons as a function of the evolution variable $(z)$. In these figures, labels $(0,1)$ and $(0,2)$ stand for the respective values of the vorticity of the two colliding solitons. The abovementioned four distinct collision scenarios have been identified within the following intervals of variation of the kick parameter $\chi$ :

(a) The two colliding solitons merge into a spinless soliton with higher velocity and energy (defined as per Eq. (5)) $E(S=0) \approx 52$ for $\chi \leq 1.4$ in the $(0,1)$ collision case, and for $\chi \leq 0.8$ in the $(0,2)$ case.

(b) The output features two spinless solitons with total energy $E \approx 104$ for $1.4<\chi \leq 1.6$ in the $(0,1)$ case, and for $0.8<\chi \leq 1.7$ in the $(0,2)$ case.

(c) The two colliding solitons merge into a spinless soliton with a lower velocity and energy $E(S=0) \approx 52$ for $1.6<\chi \leq 2.2$ in the $(0,1)$ case, and for $1.7<\chi \leq 2.2$ in the $(0,2)$ case.

(d) The output contains three spinless solitons with total energy $E \approx 156$ for $2.2<\chi \leq 6$ in the $(0,1)$ collision case, and for $2.2<\chi \leq 6$ in the $(0,2)$ case.

These generic collision scenarios are further illustrated in Figs. 6 and 7 by plots of the evolution of the field in the plane of $(t, z)$. It is noteworthy that the collision scenarios are quite similar for both cases, $(0,1)$ and $(0,2)$. A common feature of all outcomes is that the spinning soliton, although stable in isolation, is a fragile object, which never survives the collision. 

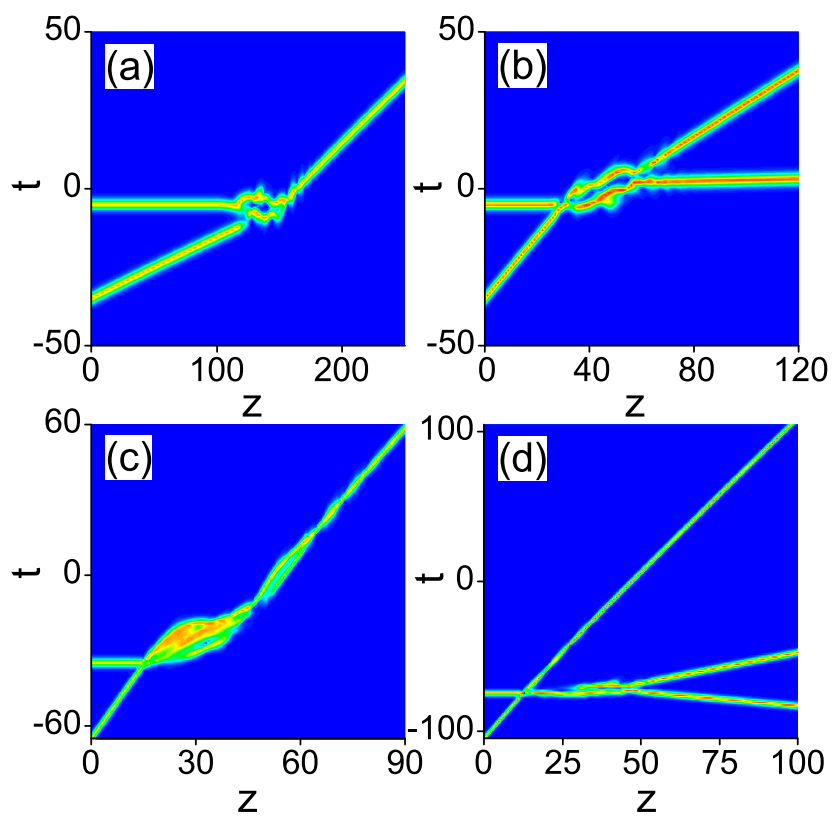

Fig. 7. (Color online) The same as in Fig. 6, but for the collision of $(0,2)$ solitons: (a) $\chi=0.2$; (b) $\chi=1$; (c) $\chi=2$; (d) $\chi=2.5$.

One can also notice that the angular momentum is not conserved in the course of the collisions - in particular, this is obvious in the case when the solitons merge into a single spinless (fundamental) one. Of course, the momentum nonconservation in the dissipative medium is not surprising.

\section{Conclusion}

In this work, we have presented results of the systematic numerical analysis of collisions between spinless and spinning stable dissipative solitons, in the framework of the complex GinzburgLandau equation, which, in particular, is a model of a 3D laser cavity. Recently, collisions were studied between pairs of solitons with identical or opposite vorticities, $(S, S)$ and $(S,-S)$. In this work, we for the first time considered the case of asymmetric pairs, $(S=0, S=1)$ and $(S=0, S=2)$. In these situations, the same generic scenario was observed with the increase of the collision momentum, $\chi$ - namely, merger into a single fundamental soliton in two intervals of values of $\chi$ (for both small and relatively large values of $\chi$ ), retrapping of the colliding solitons into a set of two fundamental ones in an intermediate interval of variation of $\chi$, and, eventually, splitting of the vortex soliton into a pair of spinless fragments at very large values of $\chi$. The analysis has been performed for co-axial configurations of the soliton pairs; a challenging issue would be to study interactions between $3 \mathrm{D}$ solitons in a more general geometric setting.

This work was supported, in part, by the Deutsche Forschungsgemeinschaft (DFG), Bonn.

\section{References}

1. I.S. Aranson, L. Kramer, Rev. Mod. Phys. 74, 99 (2002); P. Mandel, M. Tlidi, J. Opt. B: Quantum Semiclass. Opt. 6, R60 (2004); B.A. Malomed, Encyclopedia of Nonlinear Science, edited by A. Scott (Routledge, New York, 2005), p. 157

2. N. Akhmediev, A. Ankiewicz (eds.), Dissipative Solitons, Lect. Notes Phys. 661 (Springer, Berlin, 2005) 
3. S. Barland, J.R. Tredicce, M. Brambilla, L.A. Lugiato, S. Balle, M. Giudici, T. Maggipinto, L. Spinelli, G. Tissoni, T. Knodl, M. Miller, R. Jager, Nature (London) 419, 699 (2002); Z. Bakonyi, D. Michaelis, U. Peschel, G. Onishchukov, F. Lederer, J. Opt. Soc. Am. B 19, 487 (2002); E.A. Ultanir, G.I. Stegeman, D. Michaelis, C.H. Lange, F. Lederer, Phys. Rev. Lett. 90, 253903 (2003); N.N. Rosanov, S.V. Fedorov, A.N. Shatsev, Phys. Rev. Lett. 95, 053903 (2005)

4. N.N. Rosanov, Spatial Hysteresis and Optical Patterns (Springer, Berlin, 2002)

5. B.A. Malomed, D. Mihalache, F. Wise, L. Torner, J. Opt. B: Quantum Semiclass. Opt. 7, R53 (2005)

6. A.S. Desyatnikov, Y.S. Kivshar, L. Torner, Progr. Opt. 47, 291 (2005)

7. V.I. Petviashvili, A.M. Sergeev, Dokl. Akad. Nauk SSSR 276, 1380 (1984); Sov. Phys. Dokl. 29, 493 (1984)

8. B.A. Malomed, Physica D 29, 155 (1987); O. Thual, S. Fauve, J. Phys. (Paris) 49, 1829 (1988); S. Fauve, O. Thual, Phys. Rev. Lett. 64, 282 (1990); W. van Saarloos, P.C. Hohenberg, Phys. Rev. Lett. 64, 749 (1990); V. Hakim, P. Jakobsen, Y. Pomeau, Europhys. Lett. 11, 19 (1990); B.A. Malomed, A.A. Nepomnyashchy, Phys. Rev. A 42, 6009 (1990); P. Marcq, H. Chaté, R. Conte, Physica D 73, 305 (1994); N. Akhmediev, V.V. Afanasjev, Phys. Rev. Lett. 75, 2320 (1995); H.R. Brand, R.J. Deissler, Phys. Rev. Lett. 63, 2801 (1989); R.J. Deissler, H.R. Brand, Phys. Rev. Lett. 72, 478 (1994); 74, 4847 (1995); 81, 3856 (1998); V.V. Afanasjev, N. Akhmediev, J.M. Soto-Crespo, Phys. Rev. E 53, 1931 (1996); J.M. Soto-Crespo, N. Akhmediev, A. Ankiewicz, Phys. Rev. Lett. 85, 2937 (2000)

9. F. Smektala, C. Quemard, V. Couderc, A. Barthélémy, J. Non-Cryst. Solids 274, 232 (2000); G. Boudebs, S. Cherukulappurath, H. Leblond, J. Troles, F. Smektala, F. Sanchez, Opt. Commun. 219, $427(2003)$

10. C. Zhan, D. Zhang, D. Zhu, D. Wang, Y. Li, D. Li, Z. Lu, L. Zhao, Y. Nie, J. Opt. Soc. Am. B 19, 369 (2002)

11. G.S. Agarwal, S. Dutta Gupta, Phys. Rev. A 38, 5678 (1988); E.L. Falcão-Filho, C.B. de Araújo, J.J. Rodrigues, Jr., J. Opt. Soc. Am. B 24, 2948 (2007)

12. R.A. Ganeev, M. Baba, M. Morita, A.I. Ryasnyansky, M. Suzuki, M. Turu, H. Kuroda, J. Opt. A: Pure Appl. Opt. 6, 282 (2004)

13. L.-C. Crasovan, B.A. Malomed, D. Mihalache, Phys. Rev. E 63, 016605 (2001); Phys. Lett. A 289, $59(2001)$

14. P. Grelu, J.M. Soto-Crespo, N. Akhmediev, Opt. Exp. 13, 9352 (2005)

15. J.M. Soto-Crespo, P. Grelu, N. Akhmediev, Opt. Exp. 14, 4013 (2006)

16. V. Skarka, N.B. Aleksić, Phys. Rev. Lett. 96, 013903 (2006)

17. N. Akhmediev, J.M. Soto-Crespo, P. Grelu, Chaos 17, 037112 (2007)

18. J.M. Soto-Crespo, N. Akhmediev, P. Grelu, Phys. Rev. E 74, 046612 (2006)

19. D. Mihalache, D. Mazilu, F. Lederer, Y.V. Kartashov, L.-C. Crasovan, L. Torner, B.A. Malomed, Phys. Rev. Lett. 97, 073904 (2006)

20. D. Mihalache, D. Mazilu, F. Lederer, H. Leblond, B.A. Malomed, Phys. Rev. A 75, 033811 (2007); Phys. Rev. A 76, 045803 (2007); D. Mihalache, D. Mazilu, Rom. Rep. Phys. 60, 749 (2008)

21. D. Mihalache, Cent. Eur. J. Phys. 6, 582 (2008)

22. M. Soljacic, S. Sears, M. Segev, Phys. Rev. Lett. 81, 4851 (1998)

23. A.S. Desyatnikov, Y.S. Kivshar, Phys. Rev. Lett. 88, 053901 (2002)

24. Y.V. Kartashov, L.-C. Crasovan, D. Mihalache, L. Torner, Phys. Rev. Lett. 89, 273902 (2002); L.C. Crasovan, G. Molina-Terriza, J.P. Torres, L. Torner, V.M. Perez-Garcia, D. Mihalache, Phys. Rev. E 66, 036612 (2002); D. Mihalache, D. Mazilu, L.-C. Crasovan, B.A. Malomed, F. Lederer, L. Torner, Phys. Rev. E 68, 046612 (2003); L.-C. Crasovan, Y.V. Kartashov, D. Mihalache, L. Torner, Y.S. Kivshar, V.M. Perez-Garcia, Phys. Rev. E 67, 046610 (2003); D. Mihalache, D. Mazilu, L.-C. Crasovan, B.A. Malomed, F. Lederer, L. Torner, J. Opt. B: Quantum Semiclass. Opt. 6, S333 (2004)

25. D.V. Skryabin, A.G. Vladimirov, Phys. Rev. Lett. 89, 044101 (2002)

26. W.P. Zhong, L. Yi, R.H. Xie, M. Belić, G. Chen, J. Phys. B 41, 025402 (2008); Y.Q. Wang, Q. Guo, Chin. Phys. B 17, 2527 (2008); M.M. Petroski, M.S. Petrović, M.R. Belić, Opt. Commun. 279, 196 (2007); A. Fratalocchi, A. Piccardi, M. Peccianti, G. Assanto, Phys. Rev. A 75, 063835 (2007); D. Buccoliero, A.S. Desyatnikov, W. Królikowski, Y.S. Kivshar, Phys. Rev. Lett. 98, 053901 (2007); Y.J. He, B.A. Malomed, D. Mihalache, H.Z. Wang, Phys. Rev. A 77, 043826 (2008)

27. Y.J. He, B.A. Malomed, H.Z. Wang, Opt. Exp. 15, 17502 (2007)

28. D. Mihalache, D. Mazilu, F. Lederer, H. Leblond, B.A. Malomed, Phys. Rev. A 77, 033817 (2008) 
29. D. Mihalache, D. Mazilu, F. Lederer, H. Leblond, B.A. Malomed, Phys. Rev. E 78, 056601 (2008)

30. H. Sakaguchi, Physica D 210, 138 (2005)

31. E.A. Ultanir, G.I. Stegeman, C.H. Lange, F. Lederer, Opt. Lett. 29, 283 (2004)

32. J. Lega, J.V. Moloney, A.C. Newell, Phys. Rev. Lett. 73, 2978 (1994); Physica D 83, 478 (1995)

33. A. Desyatnikov, A. Maimistov, B. Malomed, Phys. Rev. E 61, 3107 (2000); D. Mihalache, D. Mazilu, L.-C. Crasovan, B.A. Malomed, F. Lederer, Phys. Rev. E 61, 7142 (2000); D. Mihalache, D. Mazilu, L.-C. Crasovan, I. Towers, A.V. Buryak, B.A. Malomed, L. Torner, J.P. Torres, F. Lederer, Phys. Rev. Lett. 88, 073902 (2002) 